\title{
Germanica
}

\section{Les Frères jurés (extrait)}

\section{Gunnar Gunnarsson}

Traducteur : Régis Boyer

\section{OpenEdition \\ Journals}

Édition électronique

URL : http://journals.openedition.org/germanica/1253

DOI : $10.4000 /$ germanica. 1253

ISSN : 2107-0784

\section{Éditeur}

Université de Lille

\section{Édition imprimée}

Date de publication : 1 décembre 1998

Pagination : 59-75

ISBN : 0984-2632F

ISSN : 0984-2632

\section{Référence électronique}

Gunnar Gunnarsson, « Les Frères jurés (extrait) », Germanica [En ligne], 23 | 1998, mis en ligne le 26 janvier 2012, consulté le 06 octobre 2020. URL : http://journals.openedition.org/germanica/1253 ; DOI : https://doi.org/10.4000/germanica.1253

Ce document a été généré automatiquement le 6 octobre 2020.

(c) Tous droits réservés 


\title{
Les Frères jurés (extrait)
}

\section{Gunnar Gunnarsson}

Traduction : Régis Boyer

\begin{abstract}
Ingolf et Leif les futurs premiers colonisateurs de l'Islande, sont encore de jeunes garçons qui habitent Dalsfjord, dans le sud de la Norvège, la ferme d'Ørn, père d'Ingolf
\end{abstract}

$\mathrm{X}$

1 Arriva à la ferme un mendiant.

2 Un vieil homme voûté, vêtu de hardes crasseuses et de peaux en loques, entra un soir dans la halle et trouva une place auprès du feu tout au bout du banc. Il resta là, à réchauffer ses doigts crochus bleuis de froid, tout en louchant à la ronde de ses yeux délavés et sournois. Assis de la sorte, sa barbe jaune où s'enchevêtraient diverses choses indéfinies, lui pendait entre les jambes. Ses cheveux gris descendaient en mèches le long de son dos. Mais c'était tout de même ses puissants sourcils que l'on remarquait le plus. Gris et touffus, ils cachaient presque les yeux lorsqu'ils s'abaissaient, et l'homme avait coutume ou bien de les lever tous les deux très haut d'un seul coup, ou bien de les soulever légèrement l'un après l'autre, ce qui donnait à son visage une expression étrangement changeante et presque animale.

On lui demanda les nouvelles qu'il avait à dire du nord, mais il ne savait que peu de choses. Dès qu'il fut évident qu'il n'était pas en état de fournir des renseignements corrects, il eut la permission de rester en paix pour se réchauffer. Une fois qu'il eut passé un moment à se chauffer les mains, il enleva les haillons qui lui couvraient les jambes et tendit les pieds vers le feu. Ces pieds étaient un spectacle rare d'ossements noueux et de saletés. Il avait l'air transi.

On lui apporta, dans une auge, quelques reliefs du repas du soir. Il prit l'auge avec un grognement, la posa sur ses genoux et entreprit de manger. Les mains ardentes, il chercha d'abord les meilleurs morceaux, agrippant la nourriture, renversant alentour le contenu de l'auge, mâchant de toute la tête. Pour manger, il savait manger. 

saisi... Et il mâchonne absolument comme quand une vache rumine. Où la! tu parles d'un vieux cochon!

7 Le mendiant vida l'auge de façon que, seuls, les os rongés restèrent. Puis il rota de bienêtre et se soulagea de ses vents. Après quoi il tomba dans un agréable assoupissement tandis que la nourriture se tassait. Il resta ainsi quelque temps, on aurait presque dit qu'il dormait. Mais soudain, il leva les sourcils, les deux en même temps, et regarda autour de soi de ses yeux délavés et ronds.

Ingolf et Leif étaient alors parvenus tout à côté de lui, ils étaient en train de le contempler minutieusement, l'un, ses jambes, l'autre, son visage. Ils avaient rarement vu rien de semblable. C'était, ma parole, une curiosité, tant par en haut que par en bas ! Il resta un moment à les regarder sans rien dire, passant de l'un à l'autre, les examinant attentivement et prenant tout son temps.

10 - Estoc et taille, dit-il enfin d'une voix bourdonnante de basse. - Quand l'estoc se brise, les exploits sont passés... Mais alors, toi, tu es là où tu dois! dit-il soudain en se tournant vers Ingolf et en lui scrutant le visage de ses yeux écarquillés.

11 Puis il rejeta la tête en arrière et émit un gros rire, comme provoqué par ses propres pensées.

12 Les garçons furent sur le point de croire qu'il divaguait. Ils restèrent immobiles, à le contempler. - Leif, bouche entr'ouverte. - Drôle de type! pensait il, et il en avait le ventre qui frémissait de plaisir.

13 Le vieux resta assis quelque temps encore, regardant soigneusement, et un rien malicieusement de l'un à l'autre. Leurs airs attentifs ne semblaient pas l'affecter.

14 - Vais-je vous raconter quelque chose? demanda-t-il enfin, de sa voix rocailleuse, en clignant de ses yeux délavés d'un air qui en disait long. - Faut-il que je vous parle du pays nouveau?

15 D'un air interrogateur, il tourna son visage aux sourcils haut levés et en passant rapidement de l'un à l'autre.

En face d'une adresse aussi directe venant de ce phénomène ridicule, les garçons se trouvèrent aussitôt un peu décontenancés. Ils se regardèrent furtivement et restèrent assis, têtes penchées et doigts tâtonnants.

17 - Bon, si vous ne daignez pas entendre, je ne daigne pas vous raconter ça non plus, grogna le vieux bougon, qui se secoua, baissa les sourcils et rentra en lui-même.

18 Les garçons levèrent la tête, le regardèrent puis se dévisagèrent, ils étaient soudain devenus curieux. Ingolf fit un signe de tête à Leif pour qu'il intervienne. Et Leif dit sans réfléchir :

20 - On écoute, vieux.

21 Le mendiant leva lentement les sourcils. Pas pour les regarder, toutefois. On eût dit qu'il les avait oubliés et qu'il n'avait pas entendu ce que Leif avait dit. Il continua de regarder devant soi, dans le feu, d'un regard lointain de ses vieux yeux si étrangement délavés. 
Norvège aux Féroé. Mais les dieux ne lui accordèrent pas bon vent, que ce soit parce qu'il avait négligé de leur faire des sacrifices ou qu'il se soit brouillé d'une autre façon avec Odin et Njord ${ }^{2}$. Ils lui envoyèrent une tempête et le firent dériver si loin vers l'ouest que, pour finir, il se crut tout près du Ginnungagab où les mers se précipitent dans Helheim ${ }^{3}$. Au lieu de cela, il arriva à un grand pays. Il gravit une haute montagne pour voir s'il pourrait trouver des signes que ce pays était habité. Mais on ne voyait nulle part de fumée non plus que d'autres signes disant que ce pays était habité par des humains. Lorsqu'il repartit de ce pays, il tomba beaucoup de neige sur les montagnes. Aussi l'appela-t-il Sneland ${ }^{4}$. Lui et ses gens dirent que c'était un bon pays. Quelque années passèrent sans que l'on n'entende plus parler de ce nouveau pays. Alors, il y eut un Suédois, il s'appelle Gardar Svavarsson et a des propriétés au Danemark, qui partit à la voile de Sjælland pour aller chercher l'héritage paternel de sa femme, dans les Hébrides. Alors qu'il doublait le Pettlandsfjord, il essuya une tempête et dériva. Lui aussi dériva vers l'ouest et arriva au nouveau pays. Il continua de cingler le long de ses côtes et découvrit que c'était une île. Il bâtit des maisons dans une baie qu'il appela Husevig ${ }^{5}$ et passa là l'hiver.

Quand il repartit au printemps suivant, la tempête détacha une barque qu'il avait en remorque. Dans cette barque il y avait un esclave qui s'appelait Natfare et une serve. Peut-être sont-ils parvenus à débarquer et se sont-ils logés sur place. Gardar loua fort ce nouveau pays. Il disait qu'il était couvert de forêts de la lande à la mer et qu'il avait des pâturages luxuriants. Il lui donna le nom de Gardarsholm ${ }^{6}$. Ce nom-là, on le conserva jusqu'à ce que Floke Vilgerdsson eut été là. Floke, qui est un puissant viking, s'équipa en Rogaland $^{7}$ pour se rendre à Gardarsholm. Il chargea son bateau dans le Smørsund. Avant de mettre à la voile, il organisa un grand sacrifice au cours duquel il 
accrut par magie la force de trois corbeaux. C'est pour cela que, depuis, on l'appelle Floke-aux- Corbeaux. Un cairn fut érigé à l'endroit où avait eu lieu ce sacrifice, on l'appelle Cairn-de-Floke. Il se trouve à la limite entre Hordaland et Rogaland. Flokeaux-Corbeaux cingla d'abord jusqu'aux Shetland et jeta l'ancre dans une crique qui reçut le nom de Crique-de-Floke. Aux Shetland, sa fille Geirhild périt dans un lac qui, ensuite, fut appelé Lac-de-Geirhild. Des Shetland, il cingla jusqu'aux Féroé où il maria l'une de ses filles. De là, il prit la haute mer, emportant ses trois corbeaux. Lorsqu'il eut navigué un jour et une nuit, il lâcha le premier corbeau. Celui-ci s'envola de l'arrière et disparut dans la direction d'où ils étaient venus. Floke fit encore un jour et une nuit de navigation. Puis il lâcha le second corbeau. Celui-ci s'envola droit en l'air puis revint au bateau. Un jour et une nuit encore, Floke navigua. Puis il lâcha le troisième corbeau. Celui-ci vola de l'avant et disparut. Et en poursuivant la navigation dans la direction où il avait disparu, ils trouvèrent le pays qu'ils cherchaient. Floke avait sur son bateau un homme qui s'appelait Faxe. Alors qu'ils arrivaient à un vaste fjord, Faxe prit la parole et dit : «Assurément, c'est un grand pays que nous avons trouvé - il y a de larges fleuves ici. » C'est pour cela que ce fjord reçut le nom de Faxemunding8. Floke-aux-Corbeaux ne s'engagea pas dans ce fjord. Il doubla un cap où se trouvait une grande montagne couverte de neige et traversa un large golfe aux nombreux récifs et îles. Il atterrit dans un fjord sur le côté nord de ce golfe, qu'il appela Vandfjord. Pour le littoral, il l'appela Bardestrand. Ce fjord était plein de poisson. A cause de ce riche butin, ils oublièrent de récolter du foin et donc, les moutons et les bovins qu'ils avaient emportés moururent en hiver par manque de fourrage. Le printemps fut passablement froid. Floke gravit une haute montagne, par un jour de printemps, il vit vers le nord un fjord tout plein de glace de haute mer. Voilà pourquoi il rebaptisa le pays et l'appela Islande9. Il voulait repartir cet été-là mais avant qu'ils aient été prêts à naviguer, ce fut l'automne et le temps se fit orageux. Floke avait sur son bateau deux paysans, Thorolf et Hærjolf. Lorsqu'ils furent enfin prêts à naviguer et qu'ils furent sortis du fjord, le vent leur arracha leur barque et dans celle-ci, il y avait Hærjolf. Celui-ci parvint à atterrir en un endroit auquel il donna son nom et qu'il appela Hærjolfshavn ${ }^{10}$. Floke-aux-Corbeaux qui ne voulait pas naviguer sans Hærjolf, revint vers la côte et amena son bateau indemne dans un fjord auquel il donna le nom de Havnefjord ${ }^{11}$. Sur un banc de sable au bord de ce fjord, ils trouvèrent une baleine échouée à terre. Hærjolf aussi avait éventé cette baleine : c'est là qu'ils se rencontrèrent. Ils appelèrent le banc de sable Hvaløre ${ }^{12}$, ainsi, il avait un nom. Ils repartirent de là et hivernèrent dans un fjord que Floke-auxCorbeaux, qui en avait assez maintenant de ce pays, ne daigna pas baptiser. L'été suivant, ils cinglèrent jusqu'à la Norvège. Lorsqu'à leur arrivée chez eux on les questionna sur ce nouveau pays, Floke n'avait que du mal à en dire. Hærjolf, en revanche, le louait avec modération, mentionnait ses avantages sans taire ses manques. Mais Thorolf raconta que le beurre dégouttait de chaque brin d'herbe, dans ce pays qu'ils avaient découvert, aussi fut-il appelé Thorolf le beurre. - Je ne vois pas davantage à vous dire de ce nouveau pays, conclut le mendiant un peu brusquement et en s'agitant, gêné - maintenant, c'est à vous de voir - !

33 Une fois qu'il se fut tu, Ingolf et Leif restèrent un moment à le regarder fixement.

34 - Et pourquoi cela? demanda enfin Ingolf. Nous n'avons pas dans l'idée de changer de lieu de résidence.

35 A cela, le vieux ne répondit rien. Leif restait pensif. Quand enfin il parla, sa voix était étouffée et absente : 

allez en entendre! nuit.

- Il pourrait quand même être amusant de voir ce pays-là un jour.

- Toi, tu le verras, grogna le vieux, et il y avait un étrange triomphe méchant dans sa grosse voix - tu le verras bien assez tôt, je veux dire !

Soudain, il s'anima, de nouveau, et sa voix devint mielleuse et insistante.

- Si vous me procurez un pichet de bière, je vais vous en raconter bien davantage vous montrerai un morceau de la toile des Nornes, ouh! ouh! Un joli morceau! Elles ont tordu les fils à un point que vous n'imaginez pas!- Apportez-moi la bière, vous

Ingolf domina alors un poids étrange et inhabituel qu'il avait dans le corps, il se leva rapidement. Son humeur avait soudain chaviré, il se sentait mal à l'aise.

- Tu vas avoir de la bière, répondit-il, sec et froid. - Mais je crois que nous en avons entendu assez pour ce soir. Viens, Leif.

Leif se leva un peu à contrecœur. Il pouvait se faire, tout de même, que le vieux en ait encore à raconter sur ce nouveau pays. Pourquoi ne pas l'entendre jusqu'à la fin, même s'il racontait des sornettes de temps en temps. Mais puisque Ingolf ne voulait pas, cela n'avait pas d'importance. Il pourrait méditer tout seul sur cette île là-bas dans la mer et discuter de plus près toutes ces choses avec Ingolf.

On apporta une cruche de bière au mendiant, il la vida par petites gorgées comme pour en épuiser tout le goût.

Puis il se coucha auprès du feu, se roula en boule et dormit. Il resta couché là toute la

Le lendemain matin, il poursuivit sa marche.

Quand Leif le chercha pour l'interroger davantage, il était parti.

Leif essaya de parler à Ingolf de ce nouveau pays. Mais Ingolf était toujours occupé à autre chose quand Leif commençait à parler de l'Islande. Leif ne se rendait pas compte que Ingolf repoussait délibérément toute conversation là-dessus.

Ingolf ne parvenait pas à se délivrer d'une certaine angoisse de voir Leif être trop absorbé par la découverte de ce pays dont leur avait parlé le mendiant. Cela aurait bien ressemblé à Leif de se mettre soudain à dresser des plans pour faire le voyage, peut-être déménager pour ce pays et s'y fixer. Il ne fallait pas que cela se produise car Leif s'obstinait facilement quand il avait eu une idée, surtout si c'était une idée un peu extraordinaire. Non, Ingolf souhaitait rester à Dalsfjord, dans la ferme de ses pères. Tout ce qui avait le goût du changement et de l'aventure, il était profondément contre, dans son for intérieur.

A sa sage façon, Ingolf amena bientôt Leif à oublier ce nouveau pays.

$\mathrm{XI}$

L'hiver tirait à sa fin.

Après Goi venait Enemaaned ${ }^{13}$ et puis - en pleine éclosion du printemps - la harpe inaugura, un jeudi sacré, le premier mois de l'été.

Dans cette région du pays, le premier jour de l'été était célébré par un grand sacrifice de trois jours et trois nuits aux Gaular. 
Ce jour-là se réunissaient à la résidence $\mathrm{du}$ jarl ${ }^{14}$ des chefs et des paysans allodiaux venant de vastes territoires, chacun avec son escorte de domestiques et chacun apportant quelques provisions ainsi que d'abondantes quantités de bière. Ceux, particulièrement, qui préparaient des expéditions vikings cet été-là, ne devaient pas rester à l'écart s'ils souhaitaient avoir chance et victoire dans leur voyage.

4 Dès le matin, très tôt, la contrée se mit à s'animer. De toutes parts, on voyait approcher de grands groupes. Le soleil scintillait sur les armes récemment fourbies et brillait sur les boucliers bariolés. Les domestiques étaient, comme d'habitude, vêtus de costumes de bure grise tissée à la maison, mais les paysans allodiaux et leurs fils arrivaient en habits magnifiques faits d'étoffes étrangères. On voyait dans chaque compagnie des manteaux rouges, bleus, verts et bariolés.

Toute la journée, des groupes continuèrent à se rassembler dans la ferme. Et cette journée-là se passa à dresser les tentes, à se préparer pour la fête, à rendre visite aux amis et connaissances, à passer des accords pour l'été et régler diverses transactions.

Le jarl Atle fut invisible ce jour-là. Seuls, ses plus proches amis et les gens qui venaient le voir pour des affaires importantes furent conduits à la chambre où il s'était caché, affairé comme il était à organiser ou à mettre de l'ordre dans ses affaires comme dans celles des autres.

Parmi les gens qui vinrent lui rendre visite ce jour-là et obtinrent audience, il y avait Ørn Bjørnulfsson. Leur entretien fut très bref et le résultat fut que le jarl Atle fit appeler son fils aîné, Haasten. Celui-ci ne passa qu'un instant chez son père. Ses frères, Hærsten et Holmsten, attendaient dehors pendant ce temps-là. Haasten ne leur dit rien de sa conversation avec son père. Et comme il ne disait rien spontanément, ses frères ne le questionnèrent pas.

5 Haasten, Hærsten et Holmsten circulaient, souhaitant la bienvenue à tout chef récemment arrivé. Ils portaient de magnifiques habits, de coûteuses armes et de précieux bijoux. Chacun des frères avait sur les épaules une longue cape de soie superbe, Haasten, une rouge, Hærsten, une bleue et Holmsten, une verte. C'étaient tous les trois de beaux hommes, grands et solidement bâtis, les cheveux blonds, de nobles traits et une allure paisible. Tels qu'ils allaient là, souhaitant la bienvenue, côte à côte, Haasten toujours à l'extrême droite, Holmsten à l'extrême gauche, peu de gens se rappelaient avoir vu d'aussi beaux hommes d'un seul coup. Ils étaient très aimés, la plupart recherchaient leur amitié, rares étaient ceux qui devenaient leurs intimes.

Parmi ces derniers, il y avait Ingolf et Leif. Haasten ne chercha absolument pas à dissimuler sa joie lorsqu'il salua les deux parents. Ce fut tout de même Ingolf en particulier qu'il entoura de sa chaude amitié. Il y admettait Leif parce qu'il était inséparable de son parent et parce qu'en dépit de tout, il lui plaisait bien, il admirait à part soi son intrépidité.

so Constamment, les trois frères revenaient à l'endroit où Ingolf et Leif surveillaient l'installation des tentes tandis que leurs pères avaient déjà disparu. Ils avaient obtenu une place d'honneur dans la halle où l'on recevait certains invités, et étaient en train de goûter au breuvage de la ferme en discutant des dernières nouvelles avec d'autres personnes animées des mêmes sentiments qu'eux.

61 Ingolf finit par remarquer que Haasten lui voulait quelque chose de particulier ce jourlà et il s'arrangea pour qu'ils se trouvent seuls un moment.

Haasten alla droit au fait. 
63 - J'apprends, Ingolf, que toi et Leif voulez vous lier, demain, de fraternité jurée. Je m'étais attendu à ce que l'on en vienne là un jour, mais cela se produit quand même un peu plus tôt que je ne me l'étais imaginé...

Ingolf l'interrompit quoiqu'il ait bien compris qu'il n'avait pas dit tout ce qu'il voulait. Il relata brièvement, mais sans rien cacher, le retour chez eux après la dernière visite aux Gaular. Il laissa entendre que Leif et lui avaient assurément besoin, tous les deux, d'être unis par des liens à toute épreuve.

65 - Tu connais Leif, conclut-il - Tu sais comme il est imprudent et a besoin de protection. Le bouclier qui le défendra aura des éraflures. Mais il lui faut un bouclier. Ce bouclier, je veux, moi, l'être.

66 - Crois-tu qu'il serait exclu que Leif ait en même temps plusieurs boucliers ? demanda calmement Haasten. Ingolf pâlit légèrement, chose qui n'échappa pas à Haasten.

68 Un moment, ils restèrent à se regarder droit en face. Il régnait un silence tellement étrange entre eux. Tous deux sentaient que maintenant, leur destin se résolvait. Finalement, Ingolf tendit la main à Haasten.

70 - Haasten, mon ami, dit-il très bas, - j'espère que nous, nous resterons toujours côte à côte tant lorsqu'il s'agira d'amitié que lorsque les armes parleront. Mais un rempart de boucliers, je crois que Leif ressentirait cela comme une prison.

71 Haasten resta là, silencieux, la main de son ami dans la sienne, à le regarder dans les yeux. Le regard de l'un et de l'autre était affligé.

72 Puis Haasten dit tranquillement :

73 - Tu as parlé, et autant dire que cela ne peut être autrement. Que chacun de nous prenne soin de ses frères. J'ai le pressentiment que de cela, il pourra être besoin.

74 Il imprima une ultime pression à la main d'Ingolf et la lâcha. Ils revinrent en silence aux tentes, où Leif conversait amicalement et joyeusement avec les frères de Haasten. Il avait sorti le couteau de Holmsten et montrait en gesticulant et avec grand enjouement comment il s'était comporté pour abattre le cheval.

75 - La ceinture est payée, Holmsten! conclut-il joyeusement. - Ton couteau qui était censé me ravir la vie, l'a sauvée. Si tu as une hache, donne m'en un coup et fais m'en cadeau après ! J'ai besoin d'une hache, mais père ne veut pas m'en offrir une. Il a peur que j'en fasse un peu trop pour éprouver son utilité, à ce qu'il dit ! J'ai essayé de lui en voler une, mais il a enfermé ses armes dans un coffre que je ne parviens pas à ouvrir.

76 Leif s'arrêta lorsque survinrent Ingolf et Haasten. Un rapide coup d'œil le convainquit que quelque chose s'était passée entre eux. Ils étaient tellement silencieux. D'un claquement, il remit le couteau au fourreau et redressa involontairement son corps dégingandé.

77 Peu après, Haasten s'éloigna avec ses frères. Haasten alla tout droit à son père.

78 - Tout est en ordre? demanda le jarl Atle.

79 - Non, je me suis ravisé, répondit Haasten qui n'avait pas envie d'éclairer complètement son père, - j'ai peur que la fraternité avec Leif Rodmarsson nous cause trop de difficultés. 
80 possible, répondit le jarl Atle. - Mais Ingolf est un homme si bon, il va hériter de grands biens. Cette famille-là a beaucoup d'amis, elle constituerait un bon soutien dans des temps agités ;

81 jurée.

82 - Peut-être, répondit sèchement le jarl. - Tu es maître de tes actes, en tout cas. Mon avis n'était qu'un avis, j'espère seulement que tu ne regretteras jamais de ne pas l'avoir suivi. sacrifier n'avaient pas été lâchés ce jour-là. Les beaux chevaux vigoureux qui allaient être offerts à Odin piaffaient impatiemment dans l'écurie. Un groupe de moutons qui devaient semblablement adoucir l'esprit du Père Universel, se reposaient, patiemment abandonnés à leur sort, dans un parc, la tête de l'un reposant sur le dos d'un autre, ruminant les derniers restes du contenu de leur estomac, n'agitant que de temps à autre les oreilles d'un air légèrement insatisfait. Des bœufs et des taureaux gras à lard qui, à une exception près, devaient également être sacrifiés en l'honneur d'Odin, mugissaient sur tous les tons et donnaient des coups de cornes aux poutres de leurs stalles. Dans une dépendance gisaient dix esclaves et malfaiteurs, les mains liées derrière le dos. Ils allaient pendiller pour entrer dans la chasse sauvage du dieu de la tempête. Aujourd'hui, c'était Odin qui recevrait les offrandes. Il y aurait pourtant aussi un petit divertissement pour Thor. Dans un coin de la dépendance où les esclaves attendaient la corde pour les pendre, gisait un ballot en haillons. C'était la serve Trude, qui avait volé, et qui, puisque, tout de même, elle allait perdre la vie, pourrait aussi bien servir d'offrande à l'auteur des fracas du tonnerre. aiguës pour les transformer en vagues contours d'ombres, les gens commencèrent à s'attrouper autour du temple. Ils avaient laissé toutes les armes sous surveillance dans leurs tentes. avant que la ferme devînt résidence de jarls. La dignité de godi ${ }^{15} \mathrm{du}$ temple était passée en héritage de père en fils depuis des temps immémoriaux et le jarl Atle le mince en avait, donc, hérité.

Le temple était un grand bâtiment spacieux, avec de grosses poutres, une entrée dans l'un des murs longitudinaux, tout près du pignon. Des flambeaux de poix, brûlants et fumants, étaient suspendus aux murs dans de gros anneaux de fer, chacun gardé par un esclave. Lorsque l'on entrait, on n'entrevoyait qu'indistinctement, dans la lumière vacillante, les dieux siégeant sur leurs socles derrière une cloison basse, à l'extrémité opposée du temple. Au delà de la cloison, le commun des mortels n'osait pénétrer, seuls le pouvaient le godi et ses acolytes consacrés à cette fin, ses assistants pour les offrandes et les sacrifices. Les dieux étaient disposés en un vaste demi-cercle. Il y en avait beaucoup, tant masculins que féminins. La plupart étaient magnifiquement vêtus, 
certains, même, parés d'anneaux d'or et de pierres précieuses. Mais l'attention était surtout attirée par les trois dieux principaux, Odin, Thor et Frey qui siégeaient au milieu du demi-cercle. Au centre trônait Thor, ici, comme en maints autres lieux de Norvège, c'était lui qui était le plus vénéré. Il était assis dans son char de tonnerre attelé de boucs peints, aux cornes d'or. Ces boucs étaient montés sur roues, prêts à être tirés pour une procession solennelle aux fêtes de Thor. Dans la dextre, il tenait son marteau à manche court, haut levé. Son allure inspirait la crainte. Juste devant lui, il y avait une mince dalle, un bord aigu vers le haut et disposée de chant.

90 A droite de Thor siégeait Odin, dans un char qui était à la fois plus grand et plus magnifique que celui de Thor, mais sans attelage. Odin était assis sur un siège décoré de runes et de signes sacrés. Il tenait dans la main une longue lance et regardait tout droit, fixement, de son œil unique.

91 A gauche de Thor siégeait Frey. Son socle était une grosse pierre couverte d'un tapis bariolé. A l'inverse des autres dieux, Frey était nu, exhibant un énorme signe de fécondité et levant haut la corne de cerf qui était sa seule arme.

$92 \mathrm{Au}$ milieu de l'espace formé par ce demi-cercle, sur un socle particulier, se tenait le récipient destiné à recevoir le sang sacrificiel, une grande vasque de pierre. S'y trouvaient les baguettes que l'on employait à brasser ce sang puis à asperger à la ronde. Sur ce socle il y avait en outre l'anneau sacré, un énorme anneau d'or orné de signes sacrés et ouvert sur lequel on prêtait tous les serments.

93 Lorsque les gens se furent rassemblés dans le temple, le jarl Atle le mince entra, suivi de ses acolytes.

94 Il portait des vêtements blancs à liserés rouges. Ses acolytes aussi étaient vêtus de blanc.

95 Lorsqu'il entra, une hache à long manche et à fer large sur l'épaule, la tête et les épaules plus haut que le plupart des personnes présentes, mince et élancé comme le tronc d'un sapin débarrassé de ses branches, il y eut un halètement dans plus d'une jeune poitrine et même de vieux vikings endurcis sentirent un léger frisson leur chatouiller le dos. En cet instant, cet homme faisait un pacte avec les dieux. Ils étaient là pour l'ineffable. Il se fit un silence de mort dans le temple.

Le jarl Atle avança dignement entre les groupes humains massés de part et d'autre de lui. A la cloison, ses acolytes s'arrêtèrent provisoirement, seul, le godi pénétra. Il se débarrassa de sa hache près du socle où se trouvait le récipient pour le sang. Puis il salua les trois dieux principaux d'une lente et digne génuflexion devant chacun d'eux, puis une encore pour les autres ensemble. Ensuite, il revint sur ses pas, marmonnant des formules conjuratrices, ôta de sa place l'anneau sacrificiel et se le passa au bras droit, saisit de la main gauche sa hache et leva le bras droit en un geste de commandement. C'était un signe pour le veilleur à la porte.

97 La plus magnifique de toutes les bêtes sacrificielles, un bœuf d'un noir de charbon, à la peau brillante et aux grandes cornes recourbées, fut amenée par des esclaves et accueillie, à la cloison, par d'autres esclaves, habillés en auxiliaires sacrificiels et consacré pour ce service.

98 Simultanément, deux des acolytes du godi s'avancèrent, levèrent le récipient sacrificiel de son socle et le déposèrent à quelque distance devant eux. 

là. Thor.

Le bœuf avait été amené sous forte résistance et en mugissant de colère. Il écumait de rage et montrait le blanc de ses yeux.

Le jarl Atle avait le pied gauche avancé et la hache levée à deux mains. Au moment précis où le bœuf se trouva à l'endroit voulu, la hache s'abattit en un coup puissant et exercé sur le cou de la bête. Celle-ci poussa un beuglement et s'effondra sur les genoux. Immédiatement, les esclaves furent sur elle, avec leurs longs couteaux. Une piqûre à la nuque, deux ou trois entailles dans les artères du cou - puis il tomba à la renverse, la vasque sacrificielle fut apporté pour que le précieux sang du sacrifice ne se perdît pas. Pendant ce temps, l'un des assistants remuait constamment le sang dans la vasque avec le rameau, afin que le sang ne se coagule pas.

Lorsque le sang du bœuf se fut déversé jusqu'à la dernière goutte dans la vasque, les assistants soulevèrent le corps mort avec des cordes et le portèrent de l'autre côté de la cloison. Là, il fut accueilli par d'autres esclaves qui le portèrent dehors et entreprirent aussitôt de le dépouiller.

Une par une, les bêtes furent avancées. Une par une, elles furent abattues et leur sang, versé dans la vasque. Mais leurs carcasses ne furent pas sorties ensuite, comme celle du bœuf. Elles furent jetées de côté, on leur permit d'attendre que le sacrifice fût terminé.

Un taureau pie fut offert à Frey. Tous les autres animaux furent offerts à Odin, le dieu du combat, afin qu'il donne chance et victoire aux expéditions vikings à venir cet été-

Pour finir, on amena l'unique sacrifice fait à Thor, la serve Trude. Deux esclaves la traînèrent jusqu'à la cloison où deux des assistants l'accueillirent et la dépouillèrent de ses haillons. L'assemblée attendait, retenant son souffle. Mais la serve Trude n'émit pas un gémissement, pas une plainte. Elle fut traînée par les cheveux devant le brandisseur de marteau, levée en l'air et son dos mince posé en travers du rebord aigu de la pierre. Le jarl Atle fit alors le signe du marteau sur l'offrande et les esclaves appuyèrent. Un incroyable cri de peur déchira l'air et mourut en un gémissement glaçant, qui se mua en un râle doux. Le dos brisé, la serve Trude gisait en travers de la pierre sacrificielle de

05 La vasque au sang sacrificiel, emplie jusqu'au bord, fut alors remise sur son socle par les assistants. Le jarl Atle ôta l'anneau sacrificiel de son bras, le frotta de sang puis le repassa à son bras. Il saisit alors le rameau et se mit à asperger de sang fumant. D'abord, il le fit sur Odin, puis sur Thor, puis sur Frey et ensuite sur chacun des dieux. Il aspergea de même de sang protecteur les murs, le plafond et le sol. Lorsqu'il en eut terminé de ce côté de la cloison, les assistants soulevèrent la vasque et, aspergeant à droite et à gauche, le jarl Atle marcha le long du temple, suivi de ses acolytes.

La vasque sacrificielle ne fut pas rapportée et remise en place avant qu'elle n'ait été vidée jusqu'à la dernière goutte.

Par là, toutefois, le rite sacrificiel n'était pas terminé.

Le char d'Odin fut sorti du temple et attelé de deux superbes chevaux blancs. Là-dessus, s'avança un esclave qui, en raison de sa taille gigantesque avait reçu cette mission, revêtu de la peau du bœuf avec ses cornes et ses sabots pendants et couverts de clochettes pendantes et tintinnabulantes.

9 La procession sacrificielle jusqu'au bosquet d'Odin s'organisa avec le jarl Atle en tête, portant la hache ensanglantée sur l'épaule. Derrière lui venait l'esclave portant la peau 
du bœuf et les sonnailles. Puis Odin dans son char, tiré par les chevaux blancs et entouré des assistants vêtus de blanc. La foule formait cortège.

Silencieusement, si bien que les craquements et les sonnailles tintinnabulantes du char résonnaient solitaires dans la nuit claire, la procession progressa jusqu'au bosquet d'Odin. Là attendaient déjà les neuf esclaves et malfaiteurs qui, une fois pendus et jouets du vent, devaient apaiser le dieu de la tempête, chacun lié à son arbre de mort.

111 Le char d'Odin fut mené en un lieu ouvert encadré de pierres pointues. N'osaient pénétrer dans ce cercle de pierres que le godi et ses assistants consacrés. Lorsque le char d'Odin fut arrivé en place et que la foule se fut rangée, les acolytes se rendirent, deux par deux, jusqu'aux victimes en attente. L'un arrangeait la corde et s'assurait qu'elle était solide, de même que la branche, l'autre défaisait les liens de la victime. L'un des esclaves se lamentait et implorait qu'on lui laisse la vie. Il ne rencontra que des regards pleins de mépris, il reçut coups de pied et horions des assistants. Comme il ne voulait pas se taire, ils lui coincèrent une pierre dans la bouche. Lorsque le jarl Atle vit que ses acolytes en avaient fini avec leurs préparatifs, il fit un signe. A l'instant même, les victimes furent pendues, toutes en même temps.

Elles n'avaient pas encore fini de frétiller qu'un bruissement résonna par la forêt. Une rafale de tempête imprima un mouvement de balancement aux corps pendillants.

Un tremblement de satisfaction méditative traversa les cœurs de l'assistance :

Odin avait accepté l'offrande !

Lentement, la procession repartit du bosquet d'Odin.

Lorsqu'elle atteignit le temple, les corps des bêtes sacrifiées avaient déjà été emportés par les esclaves pour être dépouillés et découpés.

118 Le cadavre de la serve Trude avait été emporté aussi. On l'avait plongé dans la source sacrée auprès du pignon du temple. Ce n'était pas la première victime que cette source engloutissait.

Odin fut reconduit à sa place à droite de Thor. vasque sacrificielle. Par là, la première partie de la fête du sacrifice - la nuit de l'abattage était achevée.

Les gens se rendirent à leurs tentes et se coulèrent sous leurs peaux pour dormir un peu.

Le jour précoce de printemps pointait déjà à l'est.

\section{NOTES}

1. Des divinités du Destin dans la religion Scandinave ancienne ; c'étaient des filandières, comme les Parques grecques.

2. Deux dieux majeurs dans la mythologie du Nord. 
3. Selon l'auteur, le monde de Hel (déesse des enfers) ou Helheim serait au fond du Vide-Béant (ou Ginnungagab) dont serait sorti le monde.

4. Littéralement : Pays de la Neige.

5. Islandais Husavik : Baie-des-Maisons.

6. Ilot de Gardar.

7. Une province de Norvège, comme le Hordaland dont il est question plus loin.

8. Baie-de-Faxe, islandais Faxafloi.

9. Pays-de-la-glace.

10. Le port de Hærjolf.

11. Le fjord-au-port.

12. Banc-de-sable-de-la-Baleine, islandais Hvalseyrr.

13. Goi et einmanudr (en vieux norois, ils sont ici donnés sous leur forme danisée, comme, d'ailleurs, à peu près tous les noms propres ou typiques) sont en effet les noms de deux mois situés, le premier de la mi-février à la mi-mars, le second de mi-mars à mi-avril.

14. Jarl est un titre nobiliaire très ancien dans le Nord.

15. Le prêtre-sacrificateur de cette religion. 